\title{
En mission dans le monde ouvrier, dans les années
}

\author{
1940-1970
}

Michèle Rault

\section{OpenEdition}

1 Journals

Édition électronique

URL : https://journals.openedition.org/clio/65

DOI : $10.4000 /$ clio. 65

ISSN : 1777-5299

Éditeur

Belin

Édition imprimée

Date de publication : 1 avril 2002

Pagination : 135-145

ISBN : 2-85816-620-X

ISSN : $1252-7017$

Référence électronique

Michèle Rault, «En mission dans le monde ouvrier, dans les années 1940-1970 », Clio. Histoire,

femmes et sociétés [En ligne], 15 | 2002, mis en ligne le 08 février 2005, consulté le 28 avril 2022. URL:

http://journals.openedition.org/clio/65; DOI : https://doi.org/10.4000/clio.65 


\section{En mission dans le monde ouvrier, dans les années 1940-1970}

Michèle RAULT

Quatre jeunes filles, Huguette Gamonet, Anne de Gasquet, MarieThérèse Romet, Jacqueline Sergent, se rencontrent en 1938 à FranceSténo, une école privée de secrétariat de Marseille. Huguette Gamonet, née dans un milieu catholique et cultivé, prend conscience au début de l'été 1939 qu'elle veut consacrer sa vie à Dieu. La détermination et l'intensité avec laquelle elle parle de cette décision unissent les quatre jeunes femmes, en quête d'un projet de vie. L'activité qu'elles mènent à l'œuvre de guerre et d'entraide pour les familles de soldats et de prisonniers, créée par les élèves du cours, leur donne une première orientation. Elles découvrent en effet la violence de la misère provoquée par la guerre dans les familles marseillaises. Issues de milieux bourgeois catholiques, elles prennent la mesure de la réalité de la condition ouvrière et constatent l'absence de l'Église dans les milieux populaires. L'expérience vécue durant tous ces mois leur montre que les visites à domicile, les dons en nature, les soins médicaux et l'aide juridique apportée par celles qui ont décidé de passer à cet effet une capacité en droit, sont insuffisants. Durant l'été 1940, Huguette Gamonet esquisse auprès de ses amies le projet de fonder quelque chose qui ne soit ni une œuvre de charité ni un couvent classique. Ensemble, elles renoncent à la vocation religieuse traditionnelle qu'elles avaient pensé mener dans une congrégation chez des sœurs Bénédictines, au Carmel ou auprès du Père de Foucauld. Elles pressentent qu'il leur faut 
créer un apostolat résolument nouveau, en vivant au milieu des ouvriers, à l'image du "Christ [...] vivant en communauté au milieu de ses apôtres»'.

\section{Au couvent Sainte-Marthe}

En 1943, ayant terminé leurs études, elles franchissent un premier pas, tentent de quitter leurs familles et de mener une vie commune dans un quartier ouvrier au service de tous ceux qui sont "humbles, pauvres, malheureux [...], dans une maison comme la leur, vivant de leur travail, sans œuvre extérieure». Mais leurs parents, peu préparés à un tel engagement, s'y opposent et les incitent à prendre avis et conseils auprès des autorités religieuses marseillaises. Elles rencontrent $\mathrm{Mgr}$ Blanc, vicaire général du diocèse de Marseille. Celui-ci leur demande de prendre contact avec l'abbé Coquillat, vicaire depuis 1942 à la paroisse Notre-Dame de Lourdes (Saint-Philippe) de Marseille et leur propose de renforcer le noviciat des religieuses trinitaires ${ }^{2}$ de Sainte-Marthe, une congrégation dont il a la charge pour le diocèse. Après de nombreux contacts et bien des hésitations, elles décident d'entrer, le 8 septembre 1943, au couvent SainteMarthe, la supérieure leur ayant donné l'assurance qu'à l'issue du noviciat, elles pourraient vivre en civil dans un quartier ouvrier et créer un apostolat adapté aux œuvres trinitaires. Dans ce couvent, chargées des tâches domestiques et des enfants de l'orphelinat, elles constatent l'écart entre une vie religieuse traditionnelle et celle qu'elles souhaitent avoir. Elles ont lu le livre des abbés Godin et Daniel, La France pays de mission ? ${ }^{3}$, qui les conforte dans leur idée qu'il y a de nouvelles formes à inventer pour que l'Église soit présente dans le monde ouvrier. Le 27 janvier 1944, elles ont une entrevue avec le dominicain Jacques Loew, docker depuis 1941 sur le port de Marseille qui tente de les convaincre de rejoindre la communau-

1 Les citations sont extraites du fonds d'archives de la communauté, conservé au Centre des Archives du Monde du Travail de Roubaix sous la cote 11997011.

2 L'ordre des Trinitaires, fondé en 1198, créa en 1200 un hôpital à Marseille pour accueillir les captifs chrétiens libérés des Musulmans.

3 Godin, Daniel 1943. 
té féminine qu'il est en train de mettre sur pied. Quelques jours plus tard, $\mathrm{H}$. Gamonet rencontre une nouvelle fois J. Loew dans sa paroisse de la Cabucelle. L'échange est vif. Les divergences portent sur les modalités d'intégration dans le milieu ouvrier. H. Gamonet et ses compagnes refusent de s'installer dans un quartier, en menant de front travail salarié et création de services, comme J. Loew le préconise dans son projet de résidences familiales ${ }^{4}$. Elles veulent créer, dans un cadre religieux, quelque chose de résolument neuf et différent de l'action catholique dont aucune n'a été membre. Il s'agit de «montrer à l'ouvrier que les 'curés' et les religieuses ne sont pas uniquement pour la classe riche mais pour eux et que nous savons vivre dans leur mansarde avec leur salaire, leurs habitudes». Elles envisagent d'adapter le noviciat du couvent à cet objectif en lui donnant une «forme plus intellectuelle» et aussi pratique, en allant vivre la vie ouvrière, "peut-être même au début dans une usine". C'est avec cette perspective qu'elles acceptent de prendre l'habit le 23 mars 1944, mais quelques jours plus tard, Mgr Blanc leur signifie qu'il ne tient pas la promesse qu'il leur avait fait d'adapter leur vocation aux ouvres trinitaires.

Elles choisissent alors de demander conseil aux jésuites et recherchent l'appui de Jean Duchamp, supérieur de la résidence de Marseille. Le 4 juillet 1944, elles lui font part, au cours d'un entretien au couvent, de l'incompréhension que provoque l'élaboration de leur projet. Ensemble, ils évoquent un départ du couvent mais J. Duchamp leur demande de terminer l'année de noviciat. Elles acceptent sa proposition et obtiennent par contre de travailler dans une cellule à part pour préciser ce qu'elles veulent faire. Il s'agit pour elles de vivre la vie des ouvriers en s'installant dans un «appartement HBM, en civil, sans titre officiel seulement avec l'appui spirituel d'un prêtre». Leur devise est «Ecce ancilla Domini» (Voici la Servante du Seigneur) et elles veulent être un «témoignage vivant de l'Évangile non par des paroles mais par actes». L'idée d'ouvrir un restaurant ouvrier ou celle de faire des études d'assistante sociale ou d'enseignement ménager est abandonnée assez vite. De plus en plus, elles pensent travailler en usine car elles ont la conviction que c'est le seul moyen d'être au

4 Loew 1945. 
fait des conditions de vie de la classe ouvrière.

Une nouvelle entrevue a lieu au début du mois d'octobre 1944 avec J. Duchamp qui leur propose cette fois de faire, à l'extérieur du couvent, un essai d'apostolat en milieu ouvrier, sans pour autant préjuger de sa forme définitive. Le 12 octobre 1944, elles décident donc de quitter Sainte-Marthe. Quatre jours plus tard, trois d'entre elles travaillent en usine. H. Gamonet et J. Sergent sont ouvrières chez Beauvoir, une usine de produits chimiques, A. de Gasquet est manutentionnaire dans un laboratoire de produits cosmétiques. Le 17 novembre, elles quittent un hébergement provisoire pour habiter dans un ancien entrepôt, rue de la Croix, en plein quartier populaire de Marseille.

\section{4-1952 : à Marseille, l'expérience de l'usine et du militantisme}

Cette installation donne immédiatement corps au projet. Elles décident de mener une vie communautaire, partageant salaires, organisation du quotidien, loisirs et vacances. Dès janvier 1945, l'équipe s'agrandit et entre octobre 1944 et 1952 , elle compte de quatre à neuf membres. Certaines de ces femmes s'engagent durablement dans la communauté, d'autres n'y font qu'un bref séjour ou la quittent pour se marier. Le recrutement n'est pas véritablement organisé et se fait, le plus souvent, par l'intermédiaire de jésuites. L'idée de créer, sur le modèle des congrégations, un noviciat pour accueillir les nouvelles est régulièrement évoquée mais n'aboutit pas. Elles cherchent en fait à organiser une vie spirituelle adaptée à leur situation et se fixent leurs propres règles. Elles décident ainsi de faire entre elles des vœux privés de pauvreté, de chasteté, d'obéissance et de charité. Chaque jour, un temps est consacré à la méditation et à la prière ; elles participent à la messe autant que leurs horaires de travail le permettent, se réunissent régulièrement autour de lectures spirituelles ou lors de récollections et pratiquent annuellement les exercices de Saint Ignace. J. Duchamp puis, à partir de 1949, Auguste Décisier qui lui succède à la procure de Marseille jusqu'en 1954, les encadrent. La formation religieuse est donc essentielle dans la structuration de la communauté mais elles accordent également une place importante à la connaissance de l'actualité économique, sociale et politique (étude du marxisme et de son rapport 
à la foi, du mouvement ouvrier, de l'histoire, de l'économie politique). Dans cette double formation, $\mathrm{H}$. Gamonet, désignée responsable de la communauté, joue un rôle prédominant.

Autre caractéristique de la communauté, le choix du travail salarié ouvrier reste prioritaire. En effet, ces femmes s'engagent peu dans la vie du quartier à l'exception de leur participation épisodique à des comités de quartier. Les possibilités d'insertion, telles qu'enseigner le catéchisme, s'établir dans un appartement d'un quartier ouvrier éloigné du centre-ville ou créer un dispensaire avec la JOC/F, ne sont pas menées à leur terme car ces femmes estiment que tout "désir d'efficacité visible et matérielle" est contradictoire avec leur forme de vie spirituelle. Elles sont donc OS, manœuvre ou manutentionnaire dans des secteurs d'activités très développés à Marseille, produits chimiques, savonneries et raffineries. En 1949, elles diversifient cependant quelque peu leurs activités, trois d'entre elles se forment au métier d'aide familiale qu'elles exercent, embauchées par le comité d'entreprise ou l'Aide aux mères, dans les familles ouvrières.

Cette vie d'usine, la communauté la définit comme une vie de contemplation et d'action. Très vite elles décident d'adhérer au syndicat CGT des produits chimiques et des produits alimentaires où elles deviennent déléguées du personnel et sont à l'origine de la création de sections syndicales; d'où le licenciement de certaines d'entre elles. Cependant leur engagement n'est pas inconditionnel. Leur progressive défiance face à l'emprise du Parti communiste sur la CGT et leur volonté d'essayer de trouver un terrain d'entente avec les patrons trouvent écho dans le débat provoqué par les fortes tensions syndicales qui aboutissent en avril 1948 à la création du syndicat Force Ouvrière. Celles qui travaillent à la raffinerie quittent la CGT et fondent, le 23 avril 1948, une section syndicale FO dont elles deviennent les déléguées mais, en mars 1950, elles démissionnent de FO. En effet, elles sont en profond désaccord avec une session de formation qu'elles viennent de suivre et qui s'est révélée très anticléricale et sans réel objectif de revendications. Par ailleurs, elles constatent, après l'expérience de ces deux années, que seule la CGT a une action efficace et une audience auprès des ouvriers de l'usine. Elles reprennent donc leur carte dans ce syndicat, convaincues de la nécessité de militer au 
sein de ce syndicat majoritaire pour être en accord avec le but qu'elles se sont donné de partager les conditions de vie et les revendications des ouvriers. Au mois de mai 1950, l'une d'elles, Marie-Thérèse Romet, est d'ailleurs déléguée au XXI ${ }^{e}$ congrès national de la Fédération CGT des travailleurs de l'Alimentation. Elle y retrouve le jésuite Charles Monier, ouvrier syndiqué à la raffinerie Saint-Louis, avec lequel la communauté a noué, depuis 1949, une amitié profonde faite d'expériences communes de travail et de militantisme, de récollections et d'une abondante correspondance.

Sur le plan religieux, elles veulent vivre incognito au milieu des ouvriers, sans révéler qui elles sont et sans avoir de lien avec la paroisse du quartier. C'est l'occasion de mettre à profit ce qu'elles observent pour informer les autorités religieuses de Marseille des conditions de travail des ouvriers. Elles rédigent ainsi de nombreux rapports d'usines. Le premier, daté de 1945, intitulé Une année d'expérience ouvrière est remis à Mgr Delay, évêque de Marseille, qui les reçoit à l'automne. Elles font valoir "l'énorme influence que peut avoir le service rendu avec amitié, affection, bonté et surtout égalité» et leur espoir que cette influence s'étende "par de multiples contacts". La communauté prend le nom d'“Amitiés ouvrières» à la fin de l'année 1945 puis plus tard celui de "Communautés ouvrières». Mgr Delay leur conseille de ne pas créer un groupe nouveau et de chercher à s'agréger à d'autres initiatives semblables. En janvier 1946, H. Gamonet et J. Sergent se rendent dans la banlieue de Paris, pour rencontrer Madeleine Delbrêl ${ }^{5}$ à Ivry-sur-Seine et Émilienne Josset $^{6}$ à Nogent-sur-Marne, toutes deux fondatrices de communautés de femmes laïques chrétiennes ${ }^{7}$. Les deux Marseillaises estiment leur engagement différent de celui de la première, par le choix du milieu d'insertion et aussi de la seconde, par le mode de vie religieuse. Lors d'une entrevue avec les responsables des Auxiliaires familiales et leur fondateur, le jésuite

5 Madeleine Delbrêl a créé en 1933 une équipe de femmes chrétiennes laïques qu'elle anime jusqu'à sa mort en 1964.

6 Émilienne Josset fonde en 1944 la Mission de France féminine et l'anime jusqu'en 1959.

7 Fouilloux 1992 : 160-181. 
Gustave Desbuquois, le débat porte sur la compatibilité de la vie religieuse avec la vie ouvrière. Ces rencontres les confortent dans l'idée qu'elles ont leur propre vocation, définie comme une «vie religieuse dans sa quintessence, dégagée de tout ce qui empêcherait notre pénétration ouvrière». Elles écartent donc toute idée de regroupement, attitude que l'on retrouve dans nombre de communautés de ce type telle l'équipe animée par M. Delbrêl.

Leur approche se formalise par conséquent dans une vie quotidienne d'ouvrière et de salariée mais aussi par le choix de différents contacts et engagements qui sont toujours pris individuellement et jamais au nom de la communauté. Elles participent à des réunions du Mouvement populaire des familles, à celles de l'Action catholique ouvrière (ACO), adhèrent au Mouvement de la Paix. J. Sergent est membre de la Commission exécutive des produits chimiques CGT qu'elle représente en 1951 à la Conférence nationale des femmes qui se tient à Paris. M-T. Romet y participe également pour représenter l'alimentation et, en 1952, elle fait partie d'une délégation de la CGT qui se rend en Allemagne de l'Est. A. de Gasquet et J. Romet (sœur de Marie-Thérèse, entrée dans la communauté en 1945) sont déléguées CGT au comité d'entreprise de la raffinerie Saint-Louis.

La question du statut de la communauté et de son orientation rebondit à plusieurs reprises. Une première fois, en 1947, lors d'une rencontre avec Georges Pierre-Puységur, prêtre-ouvrier dans le XIII ${ }^{c}$ arrondissement de Paris, avec lequel la communauté est en désaccord lorsqu'il lui propose de se regrouper avec les équipes de Paris ou celle de Lyon créée par Paul Magand, prêtre-ouvrier. En 1950, H. Gamonet entreprend alors de se rendre à Rome. Cette fois, il est question d'une éventuelle transformation de la communauté en institut séculier. Son séjour a par ailleurs été précédé de la rencontre, en Suisse, avec Hans Urs von Balthasar qui a quitté la Compagnie de Jésus pour fonder, avec Adrienne von Speyr, un institut séculier. À l'issue de ces consultations, les membres de la communauté, soucieuses de leur indépendance, pensent qu'il y a plus d'inconvénients que d'avantages à être reconnues officiellement par l'Église. En effet, le statut des instituts séculiers, qui les oblige à se définir par rapport à l'ins- 
titution, ne leur paraît pas adapté à la communauté qu'elles forment.

\section{3-1973 : de Paris à l'Algérie}

L'année 1953 marque un tournant dans l'histoire de la communauté. En effet plusieurs événements décident l'équipe à quitter Marseille dans un contexte de très forte tension entre les prêtres-ouvriers que $\mathrm{Mgr}$ Delay vient d'interdire dans son diocèse et les membres de l'action catholique ouvrière. Ayant participé aux réunions d'ACO, mais aussi très liées aux prêtres-ouvriers, elles se refusent à prendre parti dans un climat très tendu. D'autre part, la radicalisation de leurs engagements syndicaux provoque l'incompréhension de leurs familles, de la directrice du cours France-Sténo qui n'a cessé de vouloir contrôler leur démarche et des patrons chrétiens. Il leur est aussi de plus en plus difficile de vivre incognito. Bref, elles ont le sentiment qu'une installation à Paris leur donnera une ouverture, la possibilité de se faire connaître et d'élargir leur recrutement. Ce déménagement, encouragé par Joseph de Lorgeril, prêtreouvrier jésuite dans le XIII ${ }^{c}$ arrondissement, se fait en deux étapes. Deux femmes quittent Marseille en octobre 1953 et vivent dans le XIII ${ }^{c}$ d'em- $^{\prime}$ plois saisonniers. Un an plus tard, l'ensemble de l'équipe a déménagé à Paris. Les «filles de la rue de la Croix" deviennent les «filles de la rue de Belleville», quartier où plusieurs d'entre elles sont établies.

Entre 1954 et 1964, la communauté compte jusqu'à dix membres répartis en plusieurs petites équipes de trois à cinq membres, à Paris puis en banlieue, à Montreuil et à Bagnolet, communes à forte concentration ouvrière. En 1965, la décision est prise de vivre et de travailler au même endroit et de ne plus avoir d'insertion dans un quartier parisien. Une partie de la communauté s'établit dans la banlieue parisienne, à Ivry-surSeine. La vie communautaire s'organise autour de réunions régulières, de rencontres et de récollections avec des personnalités extérieures au groupe ainsi que de vacances en commun. À partir de 1957, elle se structure avec l'élection de la responsable pour une période de six ans. La communauté se fait connaître de Mgr Feltin, archevêque de Paris et continue d'entretenir des relations avec des jésuites, un prêtre de la Mission de Paris et la communauté de Montreuil. Elles deviennent également familières des 
écrits du jésuite Denis Vasse, psychanalyste qu'elles invitent à plusieurs de leurs sessions.

Mais leur insertion par le travail reste privilégiée. D'OS ou de manœuvres à Marseille, elles deviennent progressivement des ouvrières qualifiées (câbleuse, contrôleuse, soudeuse, conductrice de machine) et travaillent surtout dans les secteurs de la métallurgie, de l'automobile et de l'électromécanique. Leur engagement syndical à la CGT se veut un militantisme de base. Un élément déterminant pour la communauté, dans ces premières années à Paris, est leur engagement en faveur de la paix en Algérie. Elles adhèrent au comité de quartier pour la Paix, participent aux manifestations, distribuent des tracts et ont des contacts avec des militants algériens. En 1962, deux membres de la communauté sont déléguées à Évian par le comité de Belleville pour la première rencontre franco-algérienne.

Cette sensibilisation aux problèmes algériens les conduit à réfléchir à la création d'une équipe dans l'Algérie devenue indépendante. Ce projet, évoqué dès 1955, s'inscrit dans un courant qui traverse l'Église catholique dans son rapport aux pays sous-développés et s'appuie sur la publication de l'encyclique Populorum progressio. G. Piquet, née en Algérie et membre de la communauté depuis 1954, joue un rôle important dans son élaboration. De fait, le 17 juillet 1963, elle s'établit à Alger avec J. Sergent. Elles sont rejointes en avril 1964 par M-T. Romet. Là, elles se donnent comme objectif de partager la vie des Algériens par le travail (caissière, employée de bureau, secrétaire médicale) et par un réseau de relations qu'elles constituent. Deux d'entre elles sont syndiquées à l'UGTA et elles sont en contact avec les prêtres de la Mission de France en équipe à Alger jusqu’à leur retour en France en 1971.

À partir de 1973, la communauté se structure différemment ; le contexte économique a changé avec la diminution de l'offre de travail ouvrier, il y a aussi la maladie pour certaines d'entre elles et des aspirations nouvelles parmi les membres de l'équipe. La subdivision en petits groupes, depuis quelques années, avait déjà amorcée cette transformation. Tout en maintenant des rencontres régulières, ces femmes dont le parcours s'était peu à peu individualisé, vivent désormais de façon autonome 
au dépens de la vie en équipe constitutive de leur projet. Ainsi, H. Gamonet, devient salariée de l'Union sociale des ingénieurs puis occupe un poste de cadre à l'Assedic et se consacre à des séminaires, groupes de lectures et de recherches avec des analystes.

La constitution de cette communauté est l'une des expressions du front pionnier apostolique né du constat, dans les années 1930, de l'absence de l'Église dans le monde ouvrier, une situation que le contexte de la guerre n'a cessé d'aviver. Comme nombre de chrétiens de leur génération ces femmes ont expérimenté de nouvelles formes de présence en pratiquant ce que l'on a appelé l'enfouissement dans les milieux incroyants. La genèse et l'évolution de cette communauté permet de mesurer précisément l'implication féminine dans ce mouvement missionnaire qui a souvent été présenté comme globalement masculin, sans doute à cause de la médiatisation des prêtres-ouvriers. Ainsi, ces femmes ont construit une communauté à mi-chemin entre une vie religieuse et une vie laïque, sans autre modèle que celui qu'elles ont forgé à partir de leur propre expérience faite de découvertes et de ruptures. La force de leur engagement a résidé dans cette capacité à fixer leurs propres règles de fonctionnement en les adaptant constamment à l'évolution des contextes, de la guerre à la période post-conciliaire sans déroger aux principes (humanistes ?) qui les guidaient. Leur petit nombre et leur discrétion leur ont donné peu de visibilité et pourtant elles se sont octroyé un espace inédit qui ne peut être négligé dans l'analyse de la place des femmes dans l'Église et du rapport que certains de ses membres ont tenté de nouer avec la classe ouvrière.

\section{Bibliographie}

DURIEZ Bruno, FOUILLOUX Étienne, MICHEL Alain-René, MOURADIAN Georges, VIET-DEPAULE Nathalie, 2001, Chrétiens et ouvriers en France, 1937-1970, Paris, Éditions de l'Atelier..

FOUILLOUX Étienne, 1988, "Madeleine Delbrêl et la Mission", Le Supplément, $\mathrm{n}^{\circ} 173:$ 91-118. 
—, 1992, "Des chrétiens à Ivry-sur-Seine (1930-1960)», Banlieue rouge, 1920-1960, Éditions Autrement : 160-181.

-, 1995, "Femmes et catholicisme dans la France contemporaine. Aperçu historiographique», CLIO, Histoire, Femmes et Sociétés, 2 : 319-329.

-, 1998, Une Église en quête de liberté. La pensée catholique française entre le modernisme et Vatican II, Paris, Desclée de Brouwer.

GIARD Jean, 1994, Cinquante ans aux frontières de l'Église. De la Mission de France aux équipes d'Ivry, Paris, L'Harmattan.

GODIN Henri, DANIEL Yvan, 1943, La France pays de mission?, Lyon, Éditions de l'Abeille.

LOEW Jacques, 1945, Les résidentes familiales, Saint-Étienne.

PELLETIER Denis, 1997, Les catholiques en France depuis 1815, Paris.

PRÉVOTAT Jacques, 1998, Etre chrétien en France au XX' siècle de 1914 à nos jours, Paris, Le Seuil.

RAULT Michèle, VIET-DEPAULE Nathalie, 1998, "Missionnaires au travail en banlieue parisienne", Ouvriers en banlieue, $X I X^{c}-X X^{*}$, Paris, Éditions del l'Atelier : 290-341.

TRANVOUEZ Yvon, 1988, Catholiques d'abord. Approches du mouvement catholique en France $\left(X I X^{c}-X X^{c}\right)$, Paris, Éditions Ouvrières.

WATTEBLED Robert, 1990, Stratégies catholiques en monde ouvrier dans la France d'après-guerre, Paris, Éditions Ouvrières. 